\title{
Care homes learning together initiative: a collaborative approach to care home medicine
}

\author{
Authors: Peter N Robinson, ${ }^{\mathrm{A}}$ Anna Steel, ${ }^{\mathrm{A}}$ Helen Hopwood, ${ }^{\mathrm{B}}$ Loretta Chindo, ${ }^{\mathrm{C}}$ Cynthia Agbenyegah, ${ }^{\mathrm{C}}$ Anita Patel ${ }^{\mathrm{C}}$ \\ and Timothy Gluck ${ }^{\mathrm{A}}$
}

Aims

> To identify residents with challenging medical and/or psychological problems in residential care and apply principles of comprehensive geriatric assessment.

$>$ Compare this structured approach to best practice guidelines (the Framework for advanced health in care homes) ${ }^{1}$ and combine with education sessions to enhance multiprofessional learning.

\section{Methods}

Using a multiprofessional team led by a primary care physician supported by a primary care pharmacist, general practitioner (GP) trainee, specialist registrars in geriatric medicine and adult mental health services, and care home staff.

Two care homes under sole GP practice identified, and residents identified by care home staff and family invited to attend consultation.

Clinical information sought through primary and secondary care databases including dispensing information through the patients summary care record (SCR).

Two patients reviewed per 3-hour session:

> pre-evaluation discussion with multidisciplinary team followed by clinical assessment

$>$ re-convene for discussion of proposed management plan

> complete education topic and set new for following sessions.

Compare with key domains in guidelines.

\section{Results}

Five sessions attended in two care homes. Seven patients assessed to date, one requiring acute admission.

Patient demographics:

$>$ four male, three female; mean age, 81 years

$>$ mean number of comorbidities, seven

$>$ six of seven patents had confirmed dementia (the seventh

Authors: ${ }^{A}$ Royal Free NHS Foundation Trust; ${ }^{B}$ Barnet, Enfield and

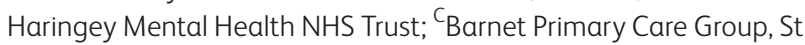
Andrews Medical Practice, London, UK. patient was too unwell for further classification).

Five educational sessions:

$>$ advanced care plans were discussed in each case with the patient and family (where available) with advanced care plans now coded as 'significant problem' in the GP database

> seven do not attempt cardiopulmonary resuscitation forms completed

> medication review in all residents with amendments made overall reduced tablet burden

> five of seven patients had a plan to change psychotropic medication

> positive feedback obtained from all clinical participants.

\section{Conclusions}

> Multidisciplinary teamworking is practical and enjoyable with educational value.

> Care home residents are a complex, frail and elderly population with a high degree of social isolation with family engagement.

> Physical and mental health assessment (including dementia) can be performed, and activity can be applied to many of the domains set out in NHS England's Framework for enhanced health in care homes ${ }^{1}$ model.

> Medications review helped to reduce inappropriate medication use.

> Discussion improved confidence and language around advanced care planning.

$>$ Telephone consultation to family proved useful.

> Shared communications via information technology solutions have limitations (especially advanced care planning) but novel workarounds can be found.

\section{Conflict of interest statement}

None declared.

\section{Reference}

1 NHS England. Framework for enhanced health in care homes. London: NHS, 2016. www.england.nhs.uk/publication/the-framework-for-enhanced-health-in-care-homes/ [Accessed 22 February 2019]. 